\title{
PREVALENCE OF CARBAPENEM RESISTANT ENTEROBACTERIACEAE- A STUDY IN A TERTIARY CARE HOSPITAL IN MUMBAI
}

\author{
Sukanya Verma1, Suyasha S. Thorat², Kishore Bisure ${ }^{3}$
}

${ }^{1}$ Speciality Medical Consultant, Department of Microbiology, Group of TB Hospital, Sewree, Mumbai, Maharashtra, India. ${ }^{2}$ Assistant Professor, Department of Microbiology, HBTMC and Dr. R. N. Cooper Hospital, Juhu, Mumbai, Maharashtra, India. ${ }_{3}^{3}$ HOD, Department of Microbiology, HBTMC and Dr. R. N. Cooper Hospital, Juhu, Mumbai, Maharashtra, India.

ABSTRACT
BACKGROUND
The global incidence of carbapenem resistant organisms is on the rise and can be attributed to indiscriminate use of carbapenem to
tackle the Extended Spectrum Beta Lactamase (ESBL) producing organisms. Carbapenem resistance is associated with resistance to
other group of antibiotics. The spread of resistance from one bug to another is not uncommon. The organism persists in the hospital
environment and is identified as cause of hospital-acquired infections. This is not only a major concern in the healthcare setting but
is also increasingly being recovered from community settings also. Limited availability of drugs to treat these infections is the biggest
concern. Identifying carbapenem resistant organisms and implementing measures to prevent the spread is need of the hour.
The aim of this study is to determine the prevalence and susceptibility pattern of Carbapenem resistant Enterobacteriaceae.

\section{MATERIALS AND METHODS}

A descriptive study was carried out in the Department of Microbiology of a tertiary care teaching institute of Mumbai. All Enterobacteriaceae isolates recovered during the six months study period of January 2017 to June 2017 were included in the study. The isolates were identified using standard conventional biochemical methods. Antimicrobial susceptibility was performed using the Kirby-Bauer disc diffusion technique and interpreted as per CLSI 2016 standards.

\section{RESULTS}

A total of 442 isolates of Enterobacteriaceae were recovered during the study period. The different strains isolated included Escherichia coli (218), Klebsiella pneumoniae (161), Proteus spp. (42), Salmonella (9), Citrobacter spp. (1) and Enterobacter spp. (1); 107 out of 442 (24.2\%) were Carbapenem resistant 18.8\% of E. coli, 37.9\% of Klebsiella pneumoniae. Maximum number of CRE were recovered from Medicine Department (34.1\%). The least number of CRE isolates were recovered from ENT (5.8\%).

\section{CONCLUSION}

The prevalence of Carbapenem resistant isolates is high in our settings. Strict adherence to infection control practices and stringent implementation of antimicrobial stewardship is essential to curb the rate of Carbapenem resistant isolates.

\section{KEY WORDS}

Enterobacteriaceae, Carbapenem Resistant, Antimicrobial Stewardship, Infection Control.

HOW TO CITE THIS ARTICLE: Verma S, Thorat SS, Bisure K. Prevalence of carbapenem resistant enterobacteriaceae- a study in a tertiary care hospital in Mumbai. J. Evolution Med. Dent. Sci. 2018;7(45):4909-4912, DOI: 10.14260/jemds/2018/1093

\section{BACKGROUND}

A rise in incidence of carbapenem resistance among Enterobacteriaceae has been observed recently.(1),(2) This rise can be attributed to increase in number of Expanded Spectrum Beta Lactamase producing organisms (ESBL). The drug of choice for these ESBL producing isolate is carbapenem. Use of carbapenem to tackle the problem of ESBL producing strains has led to indiscriminate use of carbapenem, which has ultimately resulted in developing resistance to these carbapenems.(3),(4) The other factors that may be responsible for increased incidence of CRE are the use of carbapenem in animals, their relative action and utilisation

'Financial or Other Competing Interest': None.

Submission 29-08-2018, Peer Review 18-10-2018,

Acceptance 25-10-2018, Published 05-11-2018.

Corresponding Author:

Dr. Sukanya Verma,

Speciality Medical Consultant,

Group of TB Hospital,

Jerbai Wadia Road, Sewree, Mumbai,

Maharashtra, India.

E-mail: drvermasukanya@gmail.com

DOI: $10.14260 /$ jemds $/ 2018 / 1093$

\section{(c) $(1) Ð$}

in the gut microbiota, poor isolation and infection control practices in different health care settings and increased trend of travel. Carbapenem group of drug comprises of ertapenem, imipenem, meropenem and doripenem. Resistance to carbapenem is mediated either by production of enzyme carbapenemases which are capable of hydrolysing all penicillins, cephalosporins, monobactams and carbapenem. The other mechanism of development of carbapenem resistance is poor binding of carbapenem to penicillin binding protein accompanied by overexpression of efflux pumps and overproduction of expanded spectrum beta lactamases coupled with porin loss.(5)

The resistance to carbapenem is plasmid mediated and gene responsible for carbapenemase production are found on transposons and can easily be transferred horizontally to other Enterobacteriaceae and Non-Enterobacteriaceae like Pseudomonas spp. and Acinetobacter spp. thus posing an increased risk of spread of resistance among susceptible isolates.(6) Carbapenem resistance is also associated with resistance to other antibiotic group of agents like fluoroquinolones and aminoglycosides, since the genes for resistance to these agents may be present on the same plasmid.(6) 
Increasing resistance can pose a serious threat to the health care, since these agents are one of the last resorts for treatment of multidrug resistant isolates.(5),(3) The emergence of carbapenem resistance is directly associated with raised morbidity and mortality upto 40 to $50 \%$ in some studies.(7),(8)

Though, the prevalence of multi-drug resistant organism varies from country, institution and area, the prevalence of Carbapenem resistant Enterobacteriaceae (CRE) in India ranges from 13 - 51\%.(1),(2)

Over the period of time, as more data is being collated, it is observed that these organisms are mainly isolated from the health care workers and also transmitted within. Therefore, methodologies and strategies to minimise the risk of transmissions in this group are being devised.(7)

With this background, this study was carried out to screen for the presence of Carbapenem resistant Enterobacteriaceae in our institute.

\section{MATERIALS AND METHODS}

A descriptive study was carried out in the Department of Microbiology of a tertiary care hospital of Mumbai.

The gram-negative isolates belonging to family Enterobacteriaceae that were recovered during the period of six months, i.e. from January 2017 to June 2017 were included in the study. The isolates were obtained from various clinical specimens like sputum, urine, pus and blood. The isolates were separated based on the place of isolation into outpatient and inpatient.

The isolates were identified using standard conventional biochemical testing methods. (Ref: Manual of Clinical Microbiology). After identification, the isolates were subjected to antimicrobial susceptibility testing using Kirby-Bauer disc diffusion testing. Screening for carbapenem resistance was done using meropenem $(10 \mu \mathrm{g})$ and imipenem $(10 \mu \mathrm{g})$ disc by Disc diffusion testing. The antibiotic discs were procured from Hi-Media. The zone diameters were interpreted as per CLSI 2016.

The zone diameters for Imipenem and Meropenem, if $\geq 16$ mm were considered to be sensitive, if $\leq 13 \mathrm{~mm}$ were resistant and $14 \mathrm{~mm}$ were intermediate (which were considered as resistant for final data analysis). E. coli ATCC 25922 was used as control for the Kirby-Bauer disc diffusion method.

As per CDC 2012 toolkit all isolates non-susceptible to imipenem, meropenem or doripenem and resistant to all third-generation cephalosporins tested were considered to be Carbapenem resistant Enterobacteriaceae (CRE).(9)

Later the definition was modified for surveillance purpose, so as to include Enterobacteriaceae that test as resistant to any of the carbapenem agents or evident production of carbapenemase detected by any phenotypic or molecular assay.(8)

\section{RESULTS}

A total of 442 gram-negative bacilli belonging to family Enterobacteriaceae were isolated during the study period. These isolates were recovered from various specimen that included pus, urine, blood and sputum.

Of the total 442 isolates, 273 isolates were recovered from inpatient department and 169 were recovered from outpatient department.

The different strains of gram-negative bacteria isolates included Escherichia coli (218), Klebsiella pneumoniae (161),
Proteus spp. (42), Salmonella (9), Citrobacter spp. (1) and Enterobacter (1). 107 strains out of 442 were resistant to carbapenems (24.2\%).

\begin{tabular}{|c|c|c|c|}
\hline Species & CRE & Total & Percentage \\
\hline Escherichia coli & 41 & 218 & 18.8 \\
\hline Klebsiella pneumoniae & 61 & 161 & 37.9 \\
\hline Proteus spp. & 4 & 42 & 9.5 \\
\hline Salmonella spp. & 0 & 9 & 0 \\
\hline Citrobacter spp. & 1 & 11 & 9 \\
\hline Enterobacter spp. & 0 & 1 & 0 \\
\hline Total & 107 & 442 & 24.2 \\
\hline Table 1. Species Wise distribution of Carbapenem \\
Resistant Strains \\
\hline
\end{tabular}

$18.8 \%$ of E. coli, $37.9 \%$ of Klebsiella pneumoniae, $9.5 \%$ of Proteus spp. and $9 \%$ of Enterobacter spp. were resistant to carbapenem.

\begin{tabular}{|c|c|c|c|}
\hline Specimen & CRE & Total & Percentage \\
\hline Sputum & 11 & 50 & 10.5 \\
\hline Pus & 59 & 211 & 27.96 \\
\hline Urine & 34 & 156 & 21.8 \\
\hline Blood & 3 & 25 & 12 \\
\hline Total & 107 & 442 & 24.2 \\
\hline Table 2. Specimen wise distribution of Carbapenem \\
Resistant Strains \\
\hline
\end{tabular}

$27.96 \%$ of isolates were recovered from pus, $10.5 \%$ isolates from sputum, $21.8 \%$ from urine and $12 \%$ from blood were carbapenem resistant.

\begin{tabular}{|c|c|c|c|c|c|c|c|c|}
\hline & \multicolumn{3}{|c|}{ Inpatient } & \multicolumn{3}{c|}{ 0utpatient } & $\begin{array}{c}\text { Total } \\
\text { (CRE) }\end{array}$ & \% \\
\hline & CRE & Total & \% & CRE & Total & \% & & \\
\hline Medicine & 37 & 89 & 41.5 & 7 & 40 & 17.5 & $44 / 129$ & 34.1 \\
\hline Surgery & 32 & 95 & 33.6 & 3 & 28 & 10.1 & $35 / 123$ & 28.4 \\
\hline Paediatrics & 10 & 43 & 23.25 & 1 & 24 & 4.1 & $11 / 67$ & 16.41 \\
\hline OBGY & 7 & 30 & 23.33 & 3 & 57 & 5.2 & $10 / 87$ & 11.49 \\
\hline ENT & 0 & 0 & 0 & 1 & 17 & 5.8 & $1 / 17$ & 5.8 \\
\hline Orthopaedics & 5 & 16 & 31.25 & 1 & 3 & 33 & $6 / 19$ & 31.5 \\
\hline Total & 91 & 273 & 33.33 & 16 & 169 & 9.4 & $107 / 242$ & \\
\hline Table 3. Department Wise distribution of Isolates and \\
Carbapenem Resistance \\
\hline
\end{tabular}

$33.33 \%$ and $9.4 \%$ of isolates from inpatient and outpatient isolates were carbapenem resistant respectively. Maximum number of CRE were recovered from Medicine (34.1\%) followed by Orthopaedic (31.5\%), Surgery (28.4\%), Paediatrics (16.41\%) and OBGY (11.49\%). The least number of CRE isolates were recovered from ENT (5.8\%).

\begin{tabular}{|c|c|c|c|c|}
\hline & Sensitive & Intermediate & Resistant & Total \\
\hline Imipenem & 22 & 5 & 80 & 107 \\
\hline Meropenem & 8 & 3 & 96 & 107 \\
\hline \multicolumn{5}{|c|}{ Table 4. Pattern of CRE } \\
\hline
\end{tabular}

Out of 107 CRE, 80 isolates were resistant to imipenem, 96 isolates were resistant to meropenem. 77 out of the 107 were resistant to both imipenem as well as meropenem.

5 and 3 isolates were intermediate to imipenem and meropenem respectively were included under resistant for further analysis. 


\section{DISCUSSION}

Increase in the indiscriminate use of antibiotics has led to emergence of multi-drug resistant strains. Limited treatment options are available for treating these multi-drug resistant strains. Most of the organisms carry drug resistant gene on transposon, which can easily spread from one organism to other.

Development of resistance to carbapenem is one of the burning issues that needs immediate attention and action for prevention of spread. There are various methods described by CLSI for detection of carbapenem resistance.(10) Screening for carbapenem resistance can be done using Meropenem $(10 \mu \mathrm{g})$ or Ertapenem $(10 \mu \mathrm{g})$ disc. Ertapenem is considered to be more sensitive followed by meropenem and imipenem.(8),(11) In our study, meropenem $(10 \mu \mathrm{g})$ and imipenem disc $(10 \mu \mathrm{g})$ were used for screening of carbapenem resistance.

The other methods recommended by CLSI for confirmation of carbapenem resistance include ModifiedHodge test, Carba NP (for detection of enzyme Carbapenemases), mCIM and molecular assay.(10)

Of the 442 isolates of Enterobacteriaceae recovered in our study, 107 were resistant to either one or both imipenem and meropenem. Thus, the prevalence of CRE based on this data in our study is $24.2 \%$. The prevalence of CRE varies globally. The prevalence of CRE according to some institutions in epidemic area varies between $24.7 \%$ and $29.8 \%$.(3) In a study from Mumbai, Delhi and Chandigarh the prevalence was $12.26 \%$ and $71.25 \%$ and $7.87 \%$ respectively.(12),(13),(14) The variation in prevalence of Carbapenem resistant Enterobacteriaceae from various institutions could be because of the different level of hospital infection control practises that were followed and the implementation of antimicrobial stewardship program.(14) Few studies have also detected the CRE status by surveillance of stool. The results varied from $9 \%$ to $20 \%$ in different regions of India.(15),(16)

In our study, the prevalence of Carbapenem resistance was found to be more in Klebsiella pneumoniae (61\%) followed by E. coli $(41 \%)$. This finding is supported by another study carried out by Tempe DK et al.(17),(18) In another screening study carried out, the prevalence was $26 \%$ and $41 \%$ for Klebsiella pneumoniae and E. coli respectively.(5) In studies carried out by Nagraj et al and Nair et al, the prevalence for E. coli was $60 \%$ and that for Klebsiella pneumoniae is 33\%.(1),(12)

In our study, resistance to carbapenem was also noticed in 9\% of Proteus spp. and Citrobacter species. This is the corelation with other studies.(5) None of the Enterobacter species showed Carbapenem resistance which is contrast with other studies.(5)

Carbapenem resistance in Salmonella species is not known, which is similar to the finding of our study.

Majority of the carbapenem resistant Enterobacteriaceae were isolated from the pus sample $(27 \%)$ followed by urine (21\%) sample. Other studies carried showed that CRE were more common in urine followed by pus.(5),(1),(12),(18)

The other specimen from which CRE were isolated included sputum and blood, which was almost similar to that found in other studies.(18)

$85 \%$ of the total isolates of CRE were isolated from various inpatients departments that included Medicine, Surgery,
Obstetrics and Gynaecology, Paediatric and Orthopaedics. $15 \%$ were isolated from Outpatient departments. Our study findings co-relates well with studies, which have also shown the same pattern.(19),(20)

Admission to a hospital setting predisposes the patients to colonisation followed by infection with CRE, which is the reason for increased isolation of CRE from inpatient departments.(6) Also, in the present study maximum number of isolates were recovered from the wards, which could be due to cross-transmission from one patient to another. This points out towards improving the need for implementation of various precautionary measure essential to prevent the spread of such resistant isolates. Maximum number of CRE isolates were from Medicine followed by Orthopaedics. The reason attributed to this could be use of increased usage of Carbapenem for treating invasive infections.

96 out of 107 CRE isolates were resistant to meropenem (22.39\%). 80 out of 107 CRE were resistant to Imipenem (19.2\%).

77 out of 107 were resistant to both imipenem and meropenem (17.4\%).

Increased resistance to meropenem observed in our study could be due to excessive use of meropenem as compared to imipenem. Increased resistance to meropenem is supported by other studies, which also show a similar finding.(5)

Since treatment options available for carbapenem resistant isolates are very few which include tigecycline, colistin or combination therapy increasing resistance to Carbapenem is of rising concern.

Absence of proper antimicrobial policy at our institution could have resulted in indiscriminate use of carbapenem. It has resulted in overall increase in these group of drugs. Introduction of appropriate antimicrobial stewardship program may help curb the issue. Use of optimal dose for specified duration and use of combination antibiotics are some of the measures that may play an important role.(21)

\section{CONCLUSION}

The prevalence based on screening test for Carbapenem resistance is $24 \%$. Implementation of infection control practices and proper antibiotic policy is the need of the hour to curb the rate of carbapenem resistant organism.

\section{Limitation of the Study}

The isolates in our study were screened for carbapenem resistance using disc diffusion as per CLSI. Determination of MIC that would ascertain the isolates as carbapenem resistant could not be performed due to cost constraints. Also, it could not be confirmed as to whether the isolates were resistant to carbapenem because of carbapenemases.

Main limitation of our study was that screening for carbapenem resistance was performed using meropenem and imipenem disc diffusion. However, Ertapenem which is more sensitive indicator for screening of carbapenem could not be used because of non-availability of the disc. Secondly, the confirmatory test for carbapenemase production and other confirmatory tests could not be performed because of costconstraints. 


\section{REFERENCES}

[1] Nagaraj S, Chandran SP, Shamanna P, et al. Carbapenem resistance among Escherichia coli and Klebsiella pneumoniae in a tertiary care hospital in south India. Indian J Med Microbiol 2012;30(1):93-5.

[2] Suwantarat N, Carroll KC. Epidemiology and molecular characterization of multidrug-resistant Gram-negative bacteria in Southeast Asia. Antimicrob Resist Infect Control 2016;5:15. http://www.pubmedcentral.nih.gov/articlerender.fcgi? artid $=4855802 \&$ tool=pmcentrez\&rendertype $=$ abstract

[3] Xu Y, Gu B, Huang M, et al. Epidemiology of carbapenem resistant Enterobacteriaceae (CRE) during 2000-2012 in Asia. J Thorac Dis 2015;7(3):376-85.

[4] Nordmann P, Naas T, Poirel L. Global spread of carbapenemase producing Enterobacteriaceae. Emerg Infect Dis 2011;17(10):1791-8.

[5] Parimala TV. Screening of carbapenem resistant enterobacteriaceae among nosocomial isolates: a study from south India. Indian J Curr Microb App Sci 2017;6(4):460-5.

[6] Perez F, Van Duin D. Carbapenem-resistant enterobacteriaceae: a menace to our most vulnerable patients. Cleve Clin J Med 2013;80(4):225-33.

[7] Centers for Diasease Control and Prevention, CDC. Facility Guidance for Control of Carbapenem-resistant Enterobacteriaceae (CRE). Natl Cent Emerg Zoonotic Infect Dis 2015. https://www.osha.gov/SLTC/ebola/control_prevention .html

[8] Lutgring JD, Limbago BM. The problem of carbapenemase-producing-carbapenem-resistant enterobacteriaceae detection. J Clin Microbiol 2016;54(3):529-34.

[9] Centers for Disease Control and Prevention. Guidance for control of carbapenem-resistant Enterobacteriaceae (CRE): 2012 CRE toolkit. Centers for Disease Control and Prevention, Atlanta, GA, 2012.

[10] (CLSI) C and LSI. M100 Performance standards for antimicrobial susceptibility testing. 2017: p. 282.

[11] Institute TC and LS. Performance Standards for Antimicrobial Susceptibility Testing CLSI Supplement M100S. 2014: M100-S24 p.
[12] Nair PK, Vaz MS. Prevalence of carbapenem resistant Enterobacteriaceae from a tertiary care hospital in Mumbai, India. J of Microbiology \& Infectious Diseases (JMID) 2013;3(4):207-10.

[13] Kumar S, Bahdauria S. Leading trend of carbapenem resistance in enterobacteriaceae in India. Int J Basic Appl Med Res 2014;4(2):205-8.

[14] Datta P, Gupta V, Garg S, et al. Phenotypic method for differentiation of carbapenemases in Enterobacteriaceae: study from north India. Indian J Pathol Microbiol 2012;55(3):357-60.

[15] Thacker N, Pereira N, Banavali SD, et al. Alarming prevalence of community-acquired multidrug-resistant organisms colonization in children with cancer and implications for therapy: a prospective study. Indian J Cancer 2014;51(4):442-6.

[16] Rai S, Das D, Niranjan DK, et al. Carriage prevalence of carbapenem-resistant Enterobacteriaceae in stool samples: a surveillance study. Australas Med J 2014;7(2):64-7.

[17] Tempe DK, Agarwal J, Chaudhary K, et al. Carbapenem resistance patterns in general intensive care unit of a tertiary care hospital in India. MAMC J Med Sci 2015;1(2):85-91.

http://www.mamcjms.in/text.asp?2015/1/2/85/1579 18

[18] Gunjal SP, Gunjal PN, Vanaparthi N, et al. Carbapenem resistance profile amongst Escherichia coli and Klebsiella pneumoniae in a tertiary care hospital in Ahmednagar, Maharashtra. Int J Med Res Heal Sci 2014;3(2):424-7.

http://www.indianjournals.com/ijor.aspx?target=ijor:ij mrhs\&volume $=3 \&$ issue $=2 \&$ article $=037$

[19] Gupta E, Mohanty S, Sood S, et al. Emerging resistance to carbapenems in a tertiary care hospital in north India. Indian J Med Res 2006;124(1):95-8.

[20] Mate PH, Devi KS, Devi KM, et al. Prevalence of carbapenem resistance among gram-negative bacteria in a tertiary care hospital in north-east India. IOSR J of Dental \& Med Sci (IOSR-JDMS) 2014;13(12):56-60.

[21] Bogan C, Marchaim D. The role of antimicrobial stewardship in curbing carbapenem resistance. Future Microbiol 2013;8(8):979-91. 